\title{
Space-to-Space Power Beaming Enabling High Performance Rapid Geocentric Orbit Transfer
}

\author{
John W. Dankanich*, Corinne Vassallo ${ }^{\dagger}$ and Megan Tadge* \\ NASA Marshall Space Flight Center, MSFC, AL, 35812, U.S.A.
}

The use of electric propulsion is more prevalent than ever, with industry pursuing all electric orbit transfers. Electric propulsion provides high mass utilization through efficient propellant transfer. However, the transfer times become detrimental as the $\Delta V$ transitions from nearimpulsive to low-thrust. Increasing power and therefore thrust has diminishing returns as the increasing mass of the power system limits the potential acceleration of the spacecraft. By using space-to-space power beaming, the power system can be decoupled from the spacecraft and allow significantly higher spacecraft alpha $(\mathrm{W} / \mathrm{kg})$ and therefore enable significantly higher accelerations while maintaining high performance. This project assesses the efficacy of space-to-space power beaming to enable rapid orbit transfer while maintaining high mass utilization. Concept assessment requires integrated techniques for low-thrust orbit transfer steering laws, efficient large-scale rectenna systems, and satellite constellation configuration optimization. This project includes the development of an integrated tool with implementation of IPOPT, Q-Law, and power-beaming models. The results highlight the viability of the concept, limits and paths to infusion, and comparison to state-of-the-art capabilities. The results indicate the viability of power beaming for what may be the only approach for achieving the desired transit times with high specific impulse.

\section{Nomenclature}

$=$ Area, $\mathrm{m}^{2}$

$=$ Semimajor axis

$=$ Eccentricity

$=$ Acceleration due to thrust $\left(\mathrm{m} / \mathrm{s}^{2}\right)$

$=$ Specific angular orbital momentum $\left(\mathrm{m}^{2} / \mathrm{s}\right)$

$=$ Inclination

$=$ Semilatus rectum $(\mathrm{m})$

$=$ Radial distance from central body $(\mathrm{m})$

$=$ Proximity quotient $\left(\mathrm{s}^{2}\right)$

$=$ Scaling function

$=$ Weight for orbit element œ

$=$ Orbit element (one of a, e, i, $\omega, \Omega$ )

$=$ Angle of the projection of the thrust onto the orbit plane with respect to the local horizon, positive outwards (rad)

$\beta=$ Angle between the thrust and the orbit plane, positive in the direction of the oribital angular momentum (rad)

$=$ True anomaly $(\mathrm{rad})$

$=$ Gravitational parameter of the two-body system $\left(\mathrm{m}^{3} / \mathrm{s}^{2}\right)$

$=$ Argument of periapsis (rad)

$=$ Longitude of the ascending node $(\mathrm{rad})$

()$_{x x} \quad=$ Maximum over true anomaly of the maximum over thrust angle, or giving said maximum

()$_{h} \quad=$ Component along the orbital angular momentum vector

()$_{r} \quad=$ Radially outward component

()$_{\theta} \quad=$ Circumferential component

()$_{T} \quad=$ Target value

\footnotetext{
* Mission Analyst, Technology Development and Transfer Office, ZP30, NASA MSFC.

$\dagger$ Summer Intern, ZP30, Graduate Student, University of Texas, Austin.

* Summer Intern, ZP30, Graduate Student, Purdue University.
} 


\section{Introduction}

$\mathrm{W}$ hile electric propulsion is enabling industry to maintain cost competiveness, progress is only evolutionary and is limited by the power system mass, efficiency, and cost. Solar cell efficiencies continue to increase at a reasonable cadence. New solar array packaging and deployment methods should also yield continued evolutionary progress in specific power in the near-term. Even with the trends of progress, they are only evolutionary and do not alone solve the limiting detriment to electric propulsion, long transit times.

The transit time is a function of the spacecraft acceleration and therefore specific power; power available for propulsion (converted to thrust) divided by the mass of the spacecraft. While the power system efficiency and absolute power capabilities are growing, the desired spacecraft masses are also increasing. As shown in figure 1, the trend is favorable to electric propulsion and exceeding $4 \mathrm{~W} / \mathrm{kg}$, but still necessitates relatively long transit times over chemical alternatives. The results shown in figure 2 indicate the required thrusting times departing from a geo-transfer orbit (GTO) and low earth orbit (LEO) at near-term specific powers using electric propulsion.

Figure 3 illustrates the thrusting times achievable from GTO-to-GEO and LEO-to-GEO for significantly higher specific powers. Results in figures 2 and 3 were generated using NASA's

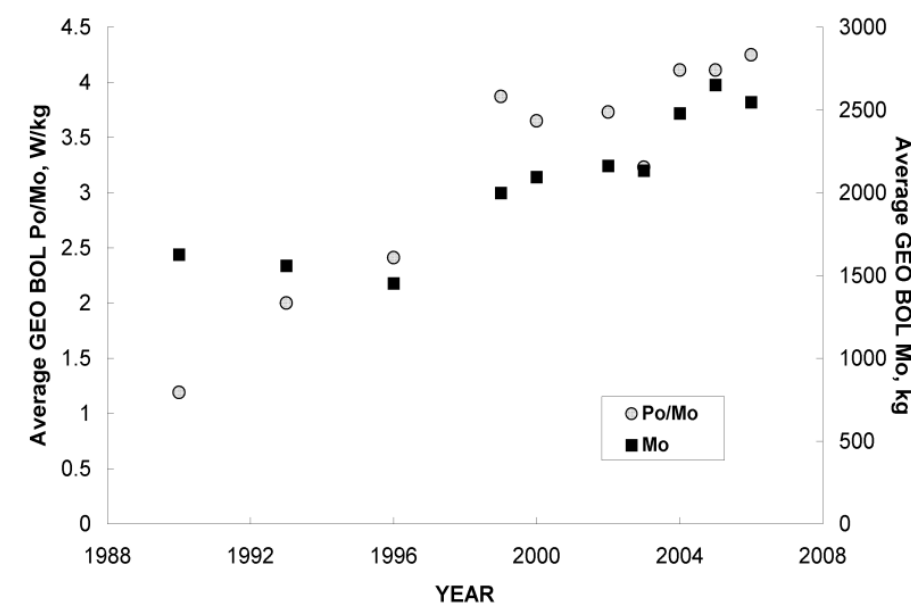

Figure 1. Trend of increasing COMSAT power and SEPSPOT tool. The transfer vehicle would still require payload power, the propulsion system, a power receiver, and thermal radiators, but a basic implementation of the power beaming spacecraft should yield an immediate leap in capability from $4 \mathrm{~W} / \mathrm{kg}$ to $40 \mathrm{~W} / \mathrm{kg}$ with a modest electric propulsion system. For a 2,000s Hall system, GTO-to-GEO transits would go from 118 days of thrusting to only 12 and LEO-to-GEO transits would decrease from 235 days to 24 days. Mass fractions from GTO and LEO would increase from $57 \%$ to $87 \%$ and $27 \%$ to $75 \%$ over chemical bipropellant respectively. If successful, the paradigm of launching large payloads to GTO for chemical insertion would likely shift to mass efficient lower cost launches to LEO.

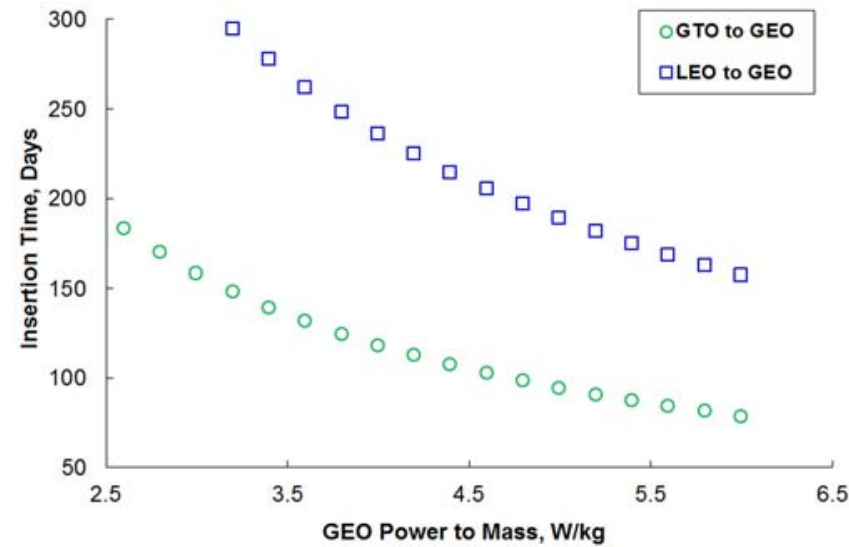

Figure 2. Near-term GTO-to-GEO transit times.

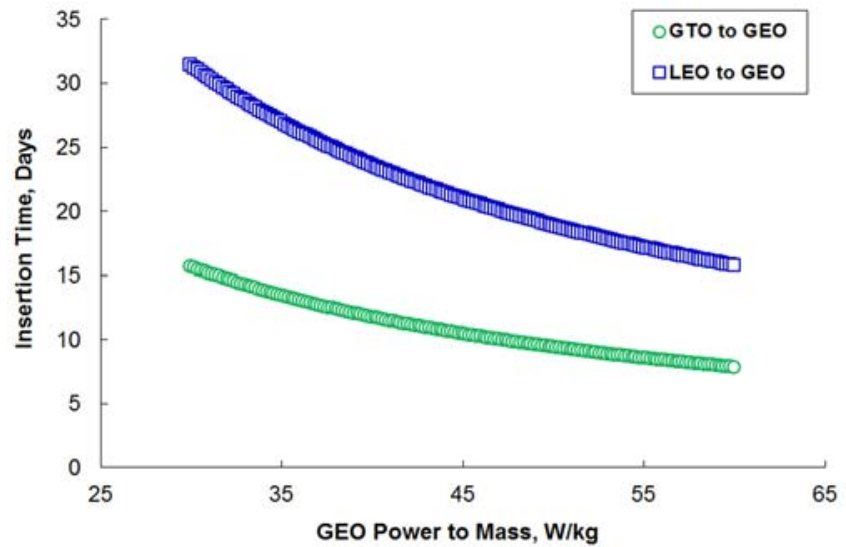

Figure 3. Transit times with SSPB.

\section{Concept Description}

The concept proposed is fundamentally high specific power $(\mathrm{W} / \mathrm{kg}$ ) electric propulsion enabled by disassociating the power generation from the transfer vehicle. The VASIMR engine made headlines with claims of orbit transfers from Earth to Mars in less than 40 days; truly revolutionary and high value to NASA. However, upon closer look; it was not the propulsion system that was revolutionary. Conventional electric propulsion thrusters can achieve 
comparable performance for the Earth-to-Mars transit provided they use the same assumption for power system specific power, $>2,000 \mathrm{~W} / \mathrm{kg}$. The specific power was revolutionary, although NASA is not investing in the required power system to achieve those goals.

One method to achieve extremely high specific power is to remove the power production from the spacecraft entirely. Power generation spacecraft can be placed in strategic orbits and beam the power to the transfer vehicle when line-of-site (LOS) exists and power generation spacecraft are illuminated. This concept is illustrated figure 4 . Note that power receiving and thermal management systems are still required on the transfer vehicle.

\section{Power Transmission Analyses}

The emphasis of the power system analyses is

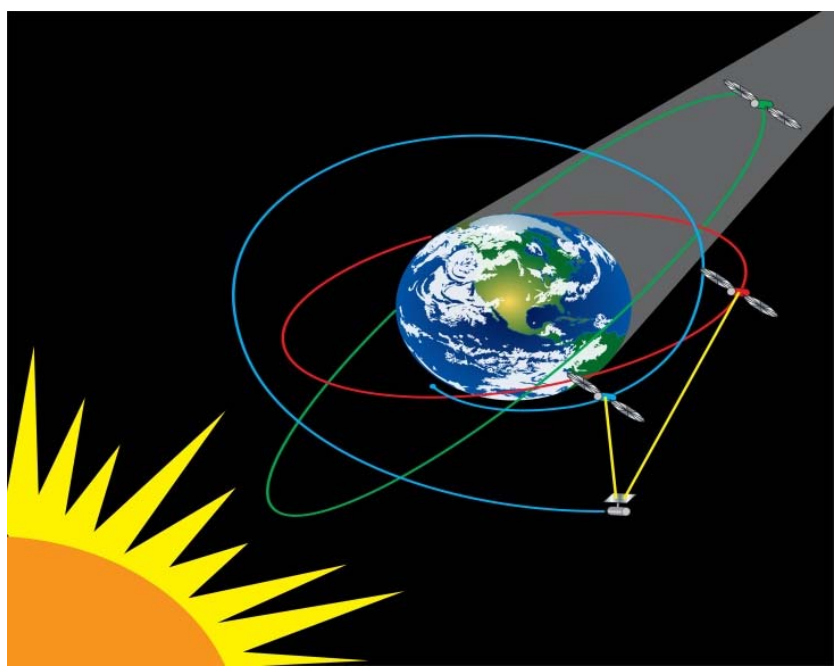

Figure 4. Illustration of space based power beaming. focused on the transfer vehicle to determine the potential power-to-mass ratios achievable for the transfer vehicle. First, power is acquired on the power-beamers through the use of solar arrays. This DC power is then converted to RF power so that it may be transmitted to the rectenna (rectifying antenna) on the transfer vehicle. A rectenna captures incident RF power and transforms it to DC power by a diode based converter. An HG filter ensures impedance match between the rectifier and the antenna for optimal power transfer. The output DC filter smoothens the output DC voltage and current by attenuating high frequency harmonics present in the RF signal or generated by the highly nonlinear rectification process ${ }^{1}$.

\section{A. Power Conversion}

The most limiting factor of the power-beaming concept is the efficiency of the power conversion process. Table 1 shows the efficiencies used for this study, based on previous GEO-to-ground wireless power transmission studies ${ }^{1,4}$. The DC-RF converter efficiency was determined by assuming the use of a Gyrotron. Gyrotrons enable high power transmission at $94 \mathrm{GHz}$ with efficiencies up to $60 \%$. Collection efficiency was based on the divergence of the beam over distance $^{2}$. Due to the large divergence of the beam, the efficiency is poor at long distances since the receiver is not large enough to capture much of the energy. Rectenna efficiencies were determined based on the state of the art efficiencies for rectenna arrays. The conversion efficiencies were scaled for $94 \mathrm{GHz}$ by calculating the effective area. As the frequency increases and the effective area of the rectenna decreases, the power density required to achieve a given efficiency increases. An efficiency curve was created based on McSpadden's and Mankins' data ${ }^{3}$ for $2.45 \mathrm{GHz}$ and then scaled to the appropriate levels for a $94 \mathrm{GHz}$ transmission. This gave the conversion efficiency based on the power density. The power density was calculated by using a series of receiver sizes to determine the power density in concentric rings within the antenna.

Though this process may seem fairly inefficient, a rectenna yields a specific power of $4000 \mathrm{~W} / \mathrm{kg}^{3}$, while the use of on-board solar arrays would yield a specific power of approximately $70-90 \mathrm{~W} / \mathrm{kg}^{4}$.

Table 1. Summary of constant efficiencies used for study.

\begin{tabular}{|l|l|l|}
\hline \multicolumn{1}{|c|}{ Sources of Inefficiency } & \multicolumn{1}{c|}{ Efficiency } & \multicolumn{1}{c|}{ Notes } \\
\hline \multicolumn{2}{|c|}{ RF Circuit Efficiency } \\
\hline DC-RF converter efficiency & 0.600 & Assumes gyrotron converter efficiency \\
\hline RF filter insertion loss (IL) & 0.891 & Estimated total IL $=0.5 \mathrm{~dB}$ \\
\hline \multicolumn{3}{|c|}{ Transmitting Antenna Efficiency } \\
\hline Subarray random electronic failures & 0.960 & Estimated 2\% failures \\
\hline Meteorite hit element failures & 1.000 & 100 failures/year \\
\hline Amplitude error & 0.996 & $\pm 1 \mathrm{~dB}$ amplitude deviation \\
\hline Phase error & 0.978 & $\pm 15^{\circ}$ phase deviation \\
\hline Antenna aperture efficiency & 0.980 & Conductive losses in aperture \\
\hline Transmitter scan loss & 1.000 & Assumes broadside radiation \\
\hline Mismatch loss & 1.000 & Assumes Array VSWR $=1.0$ \\
\hline
\end{tabular}




\begin{tabular}{|l|l|l|}
\hline \multicolumn{2}{|c|}{ Beam Coupling Efficiency } \\
\hline Collection efficiency & Varies & Depends on distance between transmitter and receiver \\
\hline Polarization loss & 1.000 & Assumes near perfect alignment \\
\hline \multicolumn{2}{|c|}{ Rectenna Efficiency } \\
\hline Rectenna random failures & 0.990 & Estimated $\%$ failures \\
\hline RF filter insertion loss (IL) & 0.891 & Estimated IL $=0.5 \mathrm{~dB}$ \\
\hline Rectenna scan loss & 1.000 & Rectenna tilted to avoid loss \\
\hline Mismatch loss & 1.000 & Assumes Array VSWR $=1.0$ \\
\hline Rectenna efficiency & Varies & Depends on distance between transmitter and receiver \\
\hline DC-DC converter efficiency & 0.950 & Assumed dc-dc converter efficiency \\
\hline System efficiency & $<41.05 \%$ & Not including collection and rectenna efficiencies \\
\hline
\end{tabular}

\section{B. System Optimization}

Figure 5 shows that as the frequency of the wireless power transmission increases, the divergence of the beam decreases. This allows for improved efficiency of the power collection with the same size antenna, or alternatively, the same efficiency while using a smaller receiver or transmitter. In this case, the size of the receiver limits the utility of this technology. Therefore that is where it would be most advantageous to reduce the size of the receiver. Increasing the frequency also improves the conversion of RF power back to DC power, since the rectenna is more efficient when operating at higher power densities.

However, use of higher frequencies

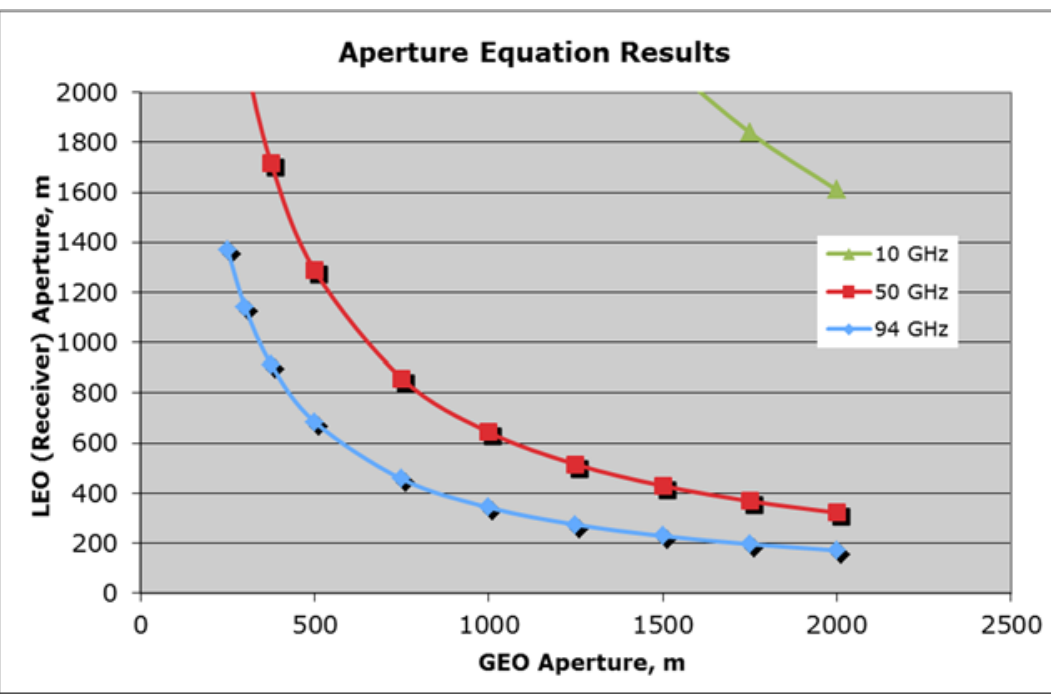

Figure 5. Aperature Equation Results; as the frequency of the wireless power transmission increases, the divergence of the beam decreases.

is not without its downsides. One limitation to higher frequencies is the decreasing efficiency of solid-state DC-to$\mathrm{RF}$ converters at higher frequencies. For example, at $4 \mathrm{GHz}$, the efficiency of a converter is $85 \%$. However, at 30 $\mathrm{GHz}$ that efficiency falls to only $65 \%$. Where they exist, alternative technologies which provide higher efficiencies at higher frequencies are currently not space ready. Another very important limitation to higher frequency transmission is the ability to build large receivers which are smooth enough to properly receive the signals. The smoothness of the receiver surface needs to be on the order of the wavelength being received. Therefore at higher frequencies, the precision needed in the surface of the receiver increases and this is limited by the size of the receiver technologies.

\section{Transmission over Large Distances.}

Over short distances, the efficiency of the rectenna and the collector can approach $100 \%$. Due to system losses and DC-to-RF efficiency, the total transmission efficiency is nearly $41 \%$. Although the rectenna conversion and collector efficiencies decrease significantly at longer distances, the distance losses can be greatly reduced by placing the power generation satellites at strategic locations. For example, in order to raise a GTO orbit to GEO, the perigee needs to be raised which requires maneuvers at apogee. Therefore, placing a power generating satellite at GEO at $14^{\circ}$ inclination will bring the satellites as close as $10,500 \mathrm{~km}$ throughout the orbit transfer which will give a power collection efficiency of $84 \%$. Therefore, the highest thrust can be achieved at optimal locations in the orbit leading to faster orbit maneuvers with only a few satellites in key locations.

\section{Performance Assessment Methodology}

There are several methods available for calculating optimal or near-optimal low thrust trajectories. The options considered for this project were Q-Law, Kluever's method, Betts' method, and FASTSTOP. Q-Law was ultimately chosen based on its ability to target all five orbital elements, allowing for use with a wider range of orbital maneuvers, 
and the ability to know that satellite's position exactly throughout the trajectory which aided in calculating the power received at the satellite. While the method used by Betts could provide an optimal solution to the problem, the optimization method is more complex to implement and should yield results within a few percent of the Q-law approach; sufficient for a preliminary concept viability study. The other two options, Kluever and FASTSTOP both use orbital averaging which results in faster trajectory simulations, but results in a loss of exact position information which is desired to assess the dependence of the orbital location of the power transmission spacecraft.

\section{A. Q-law}

Q-law is a Lyapunov feedback control law which determines the best thrust direction at the current point in the orbit based on a promity quotient $\mathrm{Q}$ which as defined as:

$$
Q=\left(1+W_{P} P\right) \sum_{o e} W_{o e} S_{o e}\left[\frac{d\left(o e, o e_{T}\right)}{\widetilde{o e}_{x x}}\right]^{2}, \quad \text { for } o e=a, e, i, \omega, \Omega
$$

where the classical orbital elements of semimajor axis, eccentricity, inclination, argument of periapsis, and longitude of the ascending node are used to target an orbit but not an exact position. This expression gives a measurement of the distance from the target orbit. The $W$ 's are weighting values, the $S$ 's are scaling functions, and $d\left(o e, o e_{T}\right)$ is a distance function indicating how far the current orbital element value is from the target value. $P$ can be used to apply a penalty function while $\widetilde{o e}_{x x}$ are analytical expressions for the maximum rate of change of the orbital elements in a given orbit.

Taking the derivative with respect to time of the quotient, $\mathrm{Q}$, gives the rate at which the orbit is approach the target orbit. This value is given by:

$$
\frac{d Q}{d t}=\sum_{o e} \frac{\partial Q}{\partial o e} \dot{o e}
$$

It is a function of the angle at which the thrust is applied since the $i e$ are Gauss's form of the variational equations for the orbit and those equations are functions of the thrust angles. Therefore, to find a near optimal low thrust orbit transfer, the time rate of change of Q is minimized all along the trajectory.

The angles $\alpha$ and $\beta$ produced during the orbit simulations matched the form of the thrust angle evolution as derived by Anastassios Petropoulos ${ }^{5}$ for the case of a GTO-to-GEO or LEO-to-GEO orbit transfer. Similar performance was also seen with most over orbit maneuvers. However, the implementation of Q-law was unable to successfully perform transfers which involved more than a $90^{\circ}$ change in inclination and could not be compared against the GTO-to-Molniya case presented by Petropoulos. The implementation of Q-law was also compared against the optimal time transfer produced by the low thrust mission design tool SEPSPOT, allowing for a rough check of the trajectory times which were approximately $10 \%$ longer with Q-law than with SEPSPOT.

\section{B. IPOPT}

IPOPT is a free optimization tool with a MATLAB interface which was used in combination with Q-Law to determine the low-thrust trajectories. Specifically, IPOPT was used to determine which angles minimized the derivative of $\mathrm{Q}$ at a specific point in the orbit. Those angles are then chosen as the thrust angles until the next optimization is performed.

IPOPT uses an interior point line search filter method to find a solution to the optimization problem. This method requires not only a calculation for the objective function, in this case $\mathrm{Q}$; which is being minimized but also the gradient and the Hessian matrix for the objective function with respect to the variables being optimized. The form is the Hessian matrix associated with this optimization problem is shown in Equation 1. If constraints are used, then those constraints and the associated Jacobian matrix also need to be provided. In the case of the angle optimization for QLaw, the angles had bounds but no constraints so no Jacobian matrix was required.

$$
H=\left[\begin{array}{ll}
\frac{\partial^{2} \dot{Q}}{\partial \alpha^{2}} & \frac{\partial^{2} \dot{Q}}{\partial \alpha \partial \beta} \\
\frac{\partial^{2} \dot{Q}}{\partial \beta \partial \alpha} & \frac{\partial^{2} \dot{Q}}{\partial \beta^{2}}
\end{array}\right]
$$




\section{Results}

Three missions were evaluated, including a GTO-to-GEO and LEO-to-GEO transfer assuming the use of the GPS constellation as power beamers and a LEO-to-GEO transfer again using the GPS constellation, but with an additional four power beamers placed in LEO.

\section{GTO-to-GEO}

The first case considered was a transfer orbit from GTO-to-GEO where the initial orbit had an apogee at $42,164 \mathrm{~km}$ and a perigee at $6,521 \mathrm{~km}$ with an inclination of $28.5^{\circ}$. For this orbit maneuver, the transfer times ranged from 7 days to over 700 days depending on the mass of the satellite, the power being transmitted and the maximum allowed received power. The results for $50 \mathrm{~kW}$ transmitters on the power generation satellites are shown in Figure 6. As expected, as satellite mass increases, transfer time increases. Furthermore, combinations of higher power transmitters and receivers both contributed to lower transfer times, however, the best transfer times were achieved when the maximum power on the transmitter and receiver were close in value. This

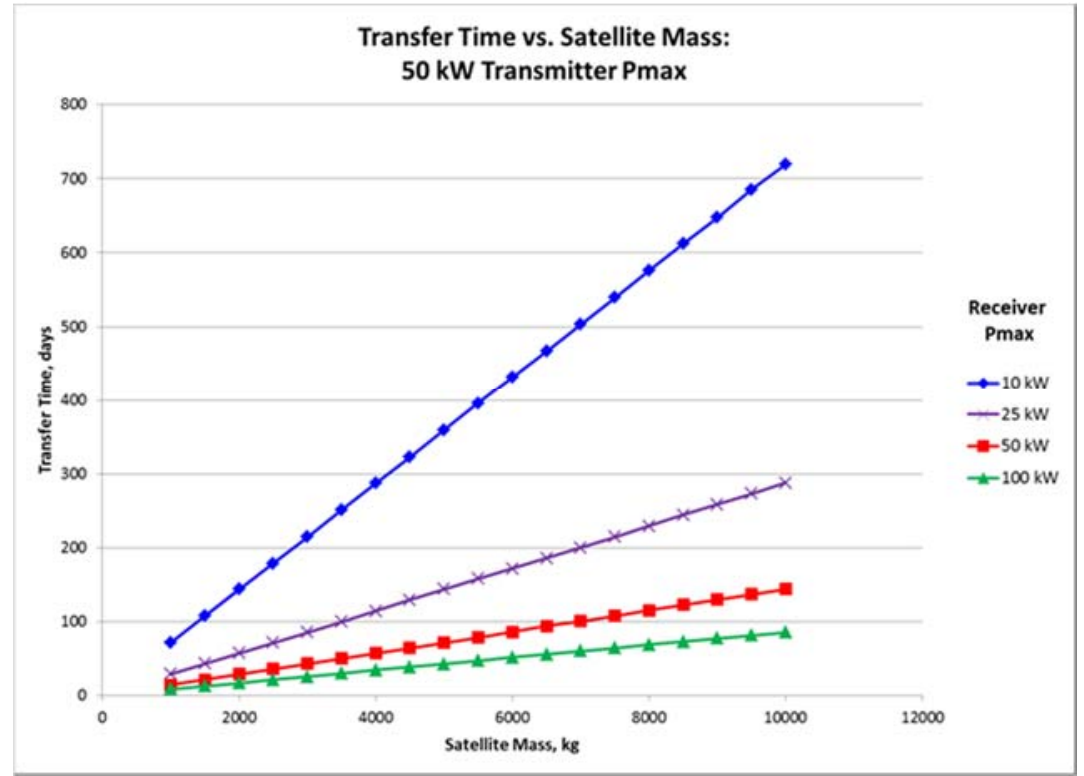

Figure 6. Transfer time versus satellite mass for $50 \mathrm{~kW}$ transmitter maximum power for select receiver maximum power for GTO-to-GEO. can additionally be seen in Figure 6, which shows transfer times for various combinations of transmitted and maximum allowed received power all assuming a satellite mass of 5,000 kg. Figure 7 also shows that when the transmitted power is less than the receiver's maximum, increasing the receiver power capibilites will have no impact on transfer time. Similarly, when receiver's maximum allowed power is less than the transmitted power, increasing the transmitted power will have little effect on transfer time since the transfer vehicle is already receiving its maximum power.

\section{LEO-to-GEO}

The second case considered a transfer orbit starting in LEO, specifically a circular orbit with a semimajor axis 6,671 $\mathrm{km}$ and an inclination of $28.5^{\circ}$. Since this starting orbit is at a lower altitude, the transfer requires a larger delta- $\mathrm{V}$ and, as expected, takes longer to complete. Figure 4 shows that the transfer times for the LEO-to-GEO cases ranged from 9 to 900 days, taking about $20 \%$ longer than the GTO-to-GEO cases.

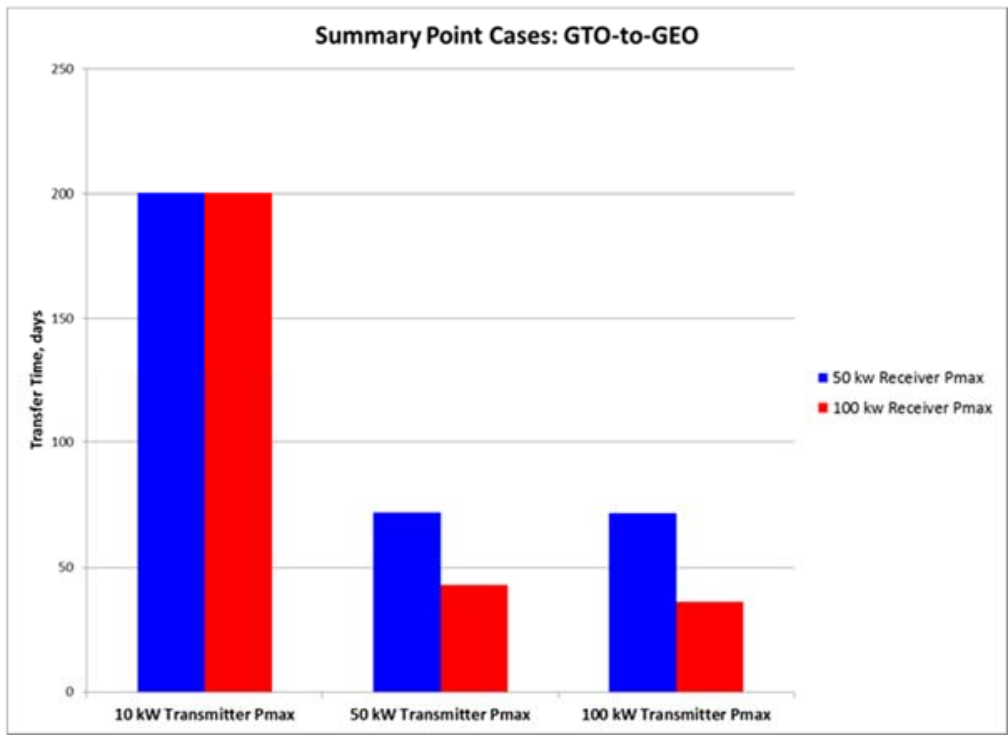

Figure 7. Summary point cases for a transfer between GTO and GEO at a satellite mass of $5,000 \mathrm{~kg}$ comparing different transmitted powers. 


\section{E. LEO-to-GEO with Additional Four Beamers}

The last case that was tested was the same transfer orbit as the LEO-toGEO transfer in Case B above, however, an additional for beamers were placed in LEO. Figure 6 shows the transfer times for this case. For both maximum allowed receiver and transmitted powers of $50 \mathrm{~kW}$, the LEO-to-GEO case with only GPS beamers yielded transfer times ranging from 18 to 185 days, where the case with the additional four beamers gave transfer times ranging from 18 to 178 days. The addition of the beamers enabled the transfer vehicle to have access to a closer power source at the start of its transfer, thus decreasing the transfer time from the GPS-beamer-only case above by about $4 \%$.

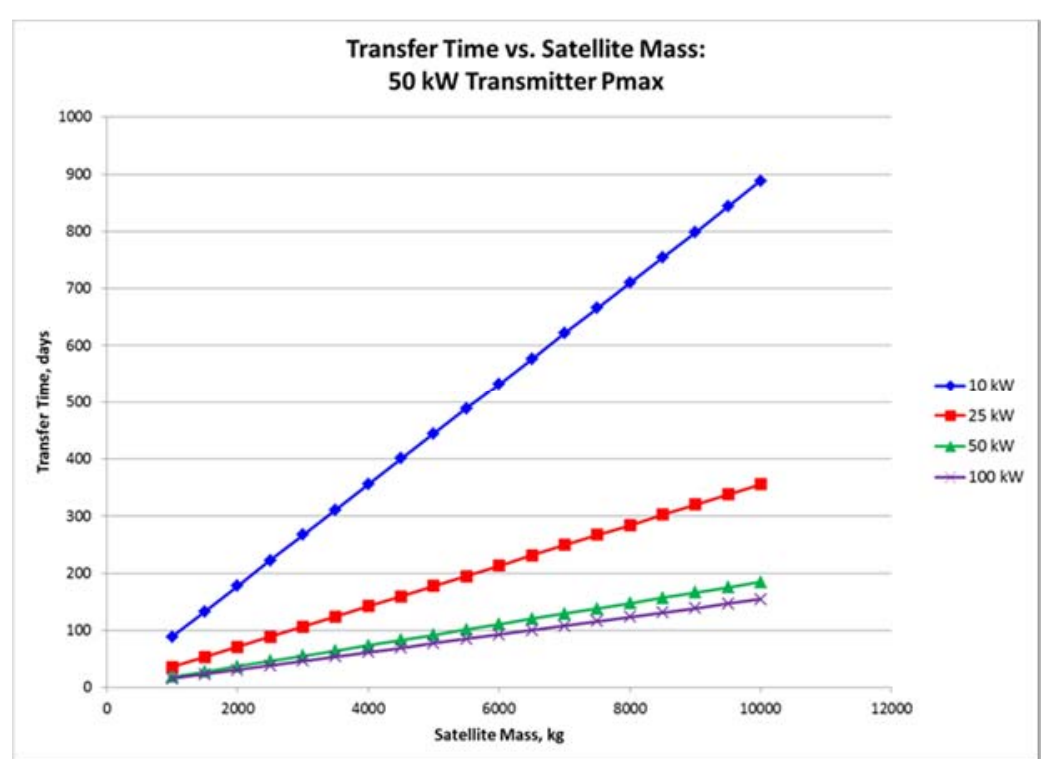

Figure 8. Transfer time versus satellite mass for a LEO to GEO transfer for $50 \mathrm{~kW}$ transmitter maximum power for select receiver maximum power.

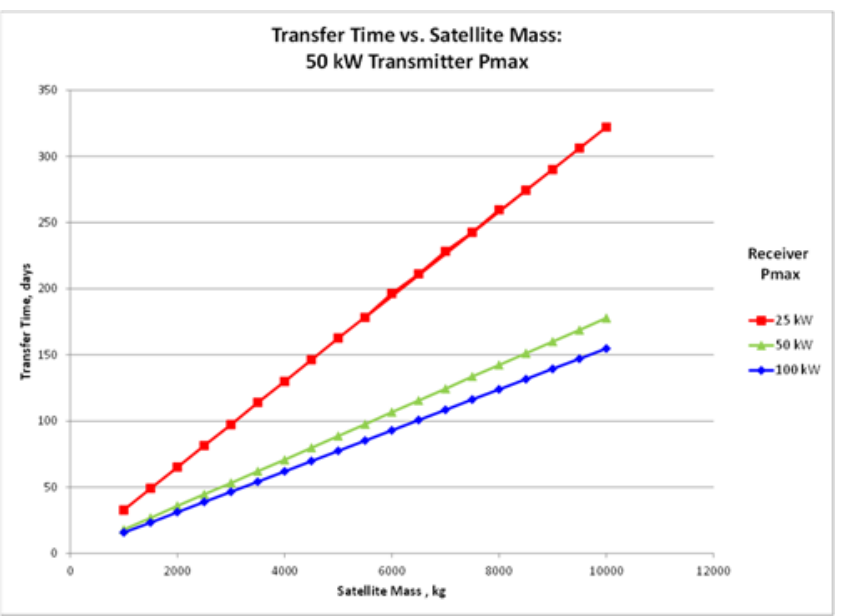

Figure 9. Transfer time versus satellite mass for $50 \mathrm{~kW}$ transmitter maximum power for select receiver maximum power.

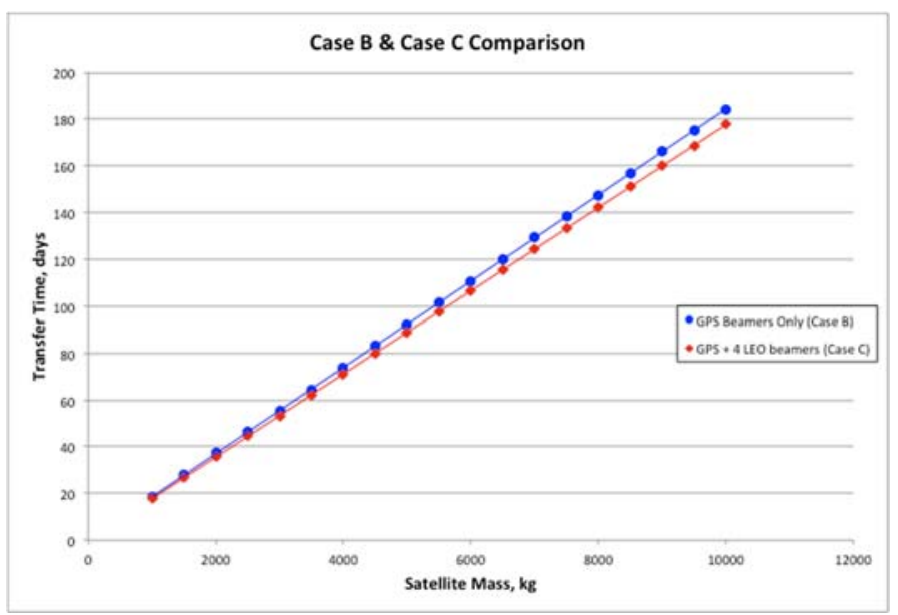

Figure 10. Transfer time versus satellite mass for two power beamer constellations both with $50 \mathrm{~kW}$ transmitter and receiver maximum power.

\section{Work to Go}

Although tests have been run using the GPS constellation as the power generation satellites, tests will also need to be performed using considering other cases such as power beaming satellites in LEO or GEO orbits. Also, although the tool currently has the capability to perform brute force optimizations for the position of consetllations of satellites, it would also be useful to implement a true optimization of the satellite positions. With the current tool, a constellation optimization was attempted, however, since optimizing the constellation requires calculating the transfer time with each position for the power generating satellites, the optimization for a GTO-to-GEO orbit transfer took 4 to 8 hours and produced values which appeared to be local, not global, minima. If the constellation optimization be performed in a timely manner, then the transfer times could likely be reduced by $10-20 \%$ if not more.

During the implementation of the power transmission between satellites, the interference between power beams coming from different satellites was not fully investigated. The power received was was assumed to add linearly. 
This is indeed the case when the beams are not phase synchronized. However, when receiving power from multiple sources if the phase of the transmitters can be synchronized, then the power received from $\mathrm{N}$ satellites can be up to $\mathrm{N}^{2}$ the power received from one satellite. Although the best case scenario occurs when the beams are exactly synchronized, most of the extra power can be attained as long as the beams are synchronized to within one-eighth of a wavelength. It may be useful to consider this effect in future space based power transmission work.

Additionally, the rectenna conversion efficiencies used with the calculation of power transmission were based on covering an entire antenna surface with the rectenna array. Considering that rectenna efficiency increases with higher power concentrations, an alternative method might be to concentrate the signal into a smaller rectenna area. This could be done by using the receiver as a reflector which sent the signal to a smaller rectenna array, much like radio telescopes use dishes to concentrate the signal. This method could be used to both increase the overall efficiency and to potentially reduce the required mass of the receiver

\section{Comments on Architecture Implementation}

This preliminary study assessment the potential to decouple the power generation system from orbit transfer vehicles to enable a dramatic improvement is spacecraft power to mass ratios beyond the current evolutionary path. One of the challenges with revolutionary concept implementation is the cost to realize return on investment. Momentum eXchange Electrodynamic Reboost (MXER) and space elevator tethers are concepts to change space transportation architectures, but require tremendous capital investments prior to first application. The use of spaceto-space power beaming, however, offers a practical infusion path. The system can be analogous to the US investment in roads. The US did not build a highway across the country as the first road. Instead, local roads were established, then intrastate and eventually interstate highway systems. It would not have been practical to begin with billions in investments in roads as an initial investment, however, the long duration investments have led to a transportation infrastructure critical to logistics of the nation. Space-to-space power beaming can similar be implementing in stepping stones. CubeSats are capable of deploying $100 \mathrm{~m}^{2}$ surfaces and a LEO power beaming system could enable CubeSat power and electric propulsion for orbit transfer and station keeping without the need for billions of spacecraft investments prior to first application. This would allow both a low cost implementation approach while closing a critical gap of SmallSat technology. Adding power beaming functionality to GEO spacecraft can be used to support GTO to GEO orbit transfer and over time, a power beaming infrastructure could evolve to as a critical asset to the logistics of geocentric space transportation.

\section{Summary}

The implementation of a geocentric power beaming architecture can revolutionize the fundamental methodology of placement and transfer of space assets. Leveraging advanced in-space propulsion is known to reduce mass required to LEO and therefore cost of access to space. Electric propulsion systems have always been power limited, and the dissociation of the power production from the transfer spacecraft can create a great leap in capability for reducing orbit transfer time; abating its limiting attribute. The power beaming architecture and infrastructure would result in a beneficial change to NASA's long-range plans and benefit nearly all space enterprise. The implementation of this concept will yield a revolutionary improvement of performance, mass reduction, and cost reduction of future spacecraft. The architecture can also enable SmallSat propulsion to augment the already expanding market by increasing capabilities and addressing the need for deorbiting spacecraft with otherwise limited onboard propulsion. This concept can leverage NASA and DoD investments in propulsion systems, large deployable arrays, advanced thermal management technologies and transmitter and receiver technologies. If fully implemented, the concept appears to be the only viable method to achieve both high mass utilization and rapid transfers from launch vehicle insertion to final operational orbits. While the concept is new relative to previous power beaming studies, it warrants further study to explore the feasibility and viability to meet entrance criteria of future investment opportunities.

\section{Acknowledgments}

The authors wish to thank the MathWorks for providing temporary licenses for Matlab and Toolboxes to support this study. The authors also wish to thank Gordon Woodcock for his support, guidance and review of work during this project. 


\section{Appendix A: Steering Law Implementation}

The control law implemented is based on Q-law. Q, which is defined as follows:

$$
Q=\left(1-W_{P} P\right) \sum_{o e} W_{o e} S_{o e}\left[\frac{d\left(o e, o e_{T}\right)}{\widetilde{o e_{x x}}}\right]^{2}, \text { for } o e=a, e, i, \omega, \Omega
$$

where classical orbital elements are used and an orbit, not a position is targeted, so true anomaly is not included. $W_{P}$ is the weighting for the penalty function which is generally either 0 or $1 . P$ is a penalty function which causes the control law to avoid certain regions, for example in this case as in Petropoulos' paper, a minimum-periapsis penalty function is used. It is defined by the following:

$$
P=e^{k\left(1-\frac{r_{p}}{r_{p m i n}}\right)}
$$

and in our case $k=100$ and $r_{p m i n}=6578 \mathrm{~km}$. $W_{o e}$ is the weight placed on driving each orbital element to its target value. For this model, all the $W_{o e}$ were set to 1 until the final orbit was reached, except in the case with no eccentricity and no inclination, when the argument of periapsis, $\omega$, and the right ascension of the ascending node, $\Omega$ are not targeted and set to $0 . S_{o e}$ is a scaling function used to prevent non-convergence. It only applies to the semimajor axis. Therefore, $S_{o e}=1$ for $o e=e, i, \omega, \Omega$. However, for the semimajor axis it is:

$$
S_{a}=\left[1+\left(\frac{a-a_{T}}{3 a_{T}}\right)^{4}\right]^{\frac{1}{2}}
$$

Finally, in the equation for $\mathrm{Q}, d\left(o e, o e_{T}\right)$ is a distance function indicating how far the current orbit is from the desired orbit. This distance function is defined as:

$$
d\left(o e, o e_{T}\right)=\left\{\begin{array}{cc}
o e-o e_{T} & \text { for oe }=a, e, i \\
\cos ^{-1}\left[\cos \left(o e-o e_{T}\right)\right] & \text { for oe }=\omega, \Omega
\end{array}\right.
$$

where the distances for $\omega$ and $\Omega$ are defined so that the shortest distance to the target will always be used.

The terms $\widetilde{o e}_{x x}$ indicate maximum value of the time derivative of the orbital element out of all thrust angles and all true anomalies. These values can be given analytically as follows:

$$
\begin{gathered}
\dot{a}_{x x}=2 f \sqrt{\frac{a^{3}(1+e)}{\mu(1-e)}} \\
\dot{i}_{x x}=\frac{2 p f}{h} \\
\dot{\Omega}_{x x}=\frac{p f}{h\left(\sqrt{1-e^{2} \cos ^{2} \omega}-e|\cos \omega|\right)} \\
\dot{\omega}_{x x i}=\frac{p f}{e h} \sqrt{p^{2} \cos ^{2} \theta_{x x}+\left(p+\cos ^{2} \omega-e|\sin \omega|\right)} \\
\dot{\omega}_{x x o}=\dot{\Omega}_{x x}|\cos i|
\end{gathered}
$$

There are two values of $\dot{\partial e_{x x}}$ for $\omega$ because it contains components in both the in-plane and out-of-plane directions. In $\widetilde{o e}_{x x}$ the in-plane and out-of-plane components are weighted in the following manner:

$$
\widetilde{\omega}_{x x}=\left(\dot{\omega}_{x x i}+0.01 \dot{\omega}_{x x o}\right) /(1.01)
$$

while for all the other orbital elements, $\widetilde{o}_{x x}=\dot{o}_{x x}$. Additionally, the values $\cos \theta_{x x}$ and $r_{x x}$ are defined as:

$$
\begin{gathered}
\cos \theta_{x x}=\left[\frac{1-e^{2}}{2 e^{3}}+\sqrt{\frac{1}{4}\left(\frac{1-e^{2}}{e^{3}}\right)^{2}+\frac{1}{27}}\right]^{\frac{1}{3}}-\left[-\frac{1-e^{2}}{2 e^{3}}+\sqrt{\frac{1}{4}\left(\frac{1-e^{2}}{e^{3}}\right)^{2}+\frac{1}{27}}\right]^{\frac{1}{3}}-\frac{1}{e} \\
r_{x x}=\frac{p}{1+e \cos \theta_{x x}}
\end{gathered}
$$

To apply Q-law, the in-plane and out-of-plane angles, $\alpha$ and $\beta$ respectively are chosen to minimize the rate of change of $\mathrm{Q}$, which is given by:

$$
\frac{d Q}{d t}=\sum_{o e} \frac{\partial Q}{\partial o e} \dot{e} e
$$

In this equation the values, $i e$, are the most straightforward, being given by Gauss's form of the variational equations, which are:

$$
\begin{gathered}
\frac{d \Omega}{d t}=\frac{r \sin (\theta+\omega)}{h \sin i} f_{h} \\
\frac{d i}{d t}=\frac{r \cos (\theta+\omega)}{h} f_{h} \\
\frac{d \omega}{d t}=\frac{1}{e h}\left[-p \cos \theta f_{r}+(p+r) \sin \theta f_{\theta}\right]-\frac{r \sin (\theta+\omega) \cos i}{h \sin i} f_{h}
\end{gathered}
$$




$$
\begin{gathered}
\frac{d a}{d t}=\frac{2 a^{2}}{h}\left(e \sin (\theta) f_{r}+\frac{p}{r} f_{\theta}\right) \\
\frac{d e}{d t}=\frac{1}{h}\left\{p \sin \theta f_{r}+[(p+r) \cos \theta+r e] f_{\theta}\right\} \\
\frac{d \theta}{d t}=\frac{h}{r^{2}}+\frac{1}{e h}\left[p \cos (\theta) f_{r}-(p+r) \sin \theta f_{\theta}\right]
\end{gathered}
$$

where $p$ is the semilatus rectum given by $p=a\left(1-e^{2}\right), h=\sqrt{\mu a\left(1-e^{2}\right)}$ is the specific orbital angular momentum, and $r=\frac{a\left(1-e^{2}\right)}{1+e \cos \theta}$ is the radius at the current position. The thrust acceleration components are given in the radial, tangential, and normal directions as follows:

$$
\begin{gathered}
f_{r}=f \cos \beta \sin \alpha \\
f_{\theta}=f \cos \beta \cos \alpha \\
f_{h}=f \sin \beta
\end{gathered}
$$

The partial derivative of $\mathrm{Q}$ with respect to the orbital elements are given by the following:

$$
\frac{\partial Q}{\partial o e}=\left\{\left(1-W_{P} P\right)\left[\sum_{o e} 2 W_{o e} \frac{\partial S_{o e}}{\partial o e} \frac{d\left(o e, o e_{T}\right)}{\widetilde{o e}_{x x}}\left(\frac{1}{\widetilde{o e}_{x x}} \frac{\partial d\left(o e, o e_{T}\right)}{\partial o e}-\frac{d\left(o e, o e_{T}\right)}{\widetilde{o e}_{x x}^{2}} \frac{\partial \widetilde{\partial e}_{x x}}{\partial o e}\right)\right]-W_{P} \frac{\partial P}{\partial o e} \sum_{o e} W_{o e} S_{o e}\left[\frac{d\left(o e, o e_{T}\right)}{\widetilde{o e}_{x x}}\right]^{2}\right\}
$$

where the sums are over $o e=a, e, i, \omega, \Omega$ and all partial derivatives in one equation are with respect to the same orbital element. Most of the partial derivatives in the above equation are trivial, however, the nonzero derivatives, $\frac{\partial \widetilde{o e}_{x x}}{\partial o e}$, can be written analytically as:

$$
\begin{aligned}
& \frac{\partial \dot{a}_{x x}}{\partial a}=3 f \sqrt{\frac{a(1+e)}{\mu(1-e)}} \\
& \frac{\partial \dot{a}_{x x}}{\partial e}=f \sqrt{\frac{a^{3}(1+e)}{\mu(1-e)}}\left(\frac{1}{1+e}+\frac{1}{1-e}\right) \\
& \frac{\partial \dot{e}_{x x}}{\partial a}=f \sqrt{\frac{1-e^{2}}{\mu a}} \\
& \frac{\partial \dot{e}_{x x}}{\partial e}=-2 e f \sqrt{\frac{a}{\mu\left(1-e^{2}\right)}} \\
& \frac{\partial i_{x x}}{\partial a}=\frac{f}{2} \sqrt{\frac{1-e^{2}}{\mu a}} \frac{1}{\sqrt{1-e^{2} \sin ^{2} \omega}-e|\cos \omega|} \\
& \frac{\partial i_{x x}}{\partial e}=f \sqrt{\frac{a}{\mu\left(1-e^{2}\right)^{3}}}\left(|\cos \omega|+\frac{e \cos ^{2} \omega}{\sqrt{1-e^{2} \sin ^{2} \omega}}\right) \\
& \frac{\partial i_{x x}}{\partial \omega}=-e f \sqrt{\frac{a}{\mu\left(1-e^{2}\right)}}\left[\frac{e \sin \omega \cos \omega}{\sqrt{1-e^{2} \sin ^{2} \omega}}+\sin (\omega) \operatorname{sgn}(\cos \omega)\right] \\
& \frac{\partial \dot{\Omega}_{x x}}{\partial a}=\frac{f}{2 \sin i} \sqrt{\frac{1-e^{2}}{\mu a}} \frac{1}{\sqrt{1-e^{2} \cos ^{2} \omega}-e|\sin \omega|} \\
& \frac{\partial \dot{\Omega}_{x x}}{\partial e}=\frac{f}{\sin i} \sqrt{\frac{a}{\mu\left(1-e^{2}\right)}} \frac{1}{\sqrt{1-e^{2} \cos ^{2} \omega}-e|\sin \omega|}\left[\frac{1-e^{2}}{\sqrt{1-e^{2} \cos ^{2} \omega}-e|\sin \omega|}\left(\frac{e \cos ^{2} \omega}{\sqrt{1-e^{2} \cos ^{2} \omega}}+|\sin \omega|\right)-e\right] \\
& \frac{\partial \dot{\Omega}_{x x}}{\partial i}=-f \sqrt{\frac{a\left(1-e^{2}\right)}{\mu}} \frac{1}{\left(\sqrt{1-e^{2} \cos ^{2} \omega}-e|\sin \omega|\right) \sin i \tan i} \\
& \frac{\partial \dot{\Omega}_{x x}}{\partial \omega}=\frac{f}{\sin i} \sqrt{\frac{a\left(1-e^{2}\right)}{\mu}} \frac{1}{\left(\sqrt{1-e^{2} \cos ^{2} \omega}-e|\sin \omega|\right)^{2}}\left(e \cos (\omega) \operatorname{sgn}(\sin \omega)-\frac{e^{2} \cos \omega \sin \omega}{\sqrt{1-e^{2} \cos ^{2} \omega}}\right) \\
& \frac{\partial \dot{\omega}_{x x i}}{\partial a}=\frac{f}{2 e} \sqrt{\frac{1-e^{2}}{\mu a}\left[1+\frac{2\left(1-\cos ^{2} \theta_{x x}\right)}{1-e \cos \theta_{x x}}+\frac{1-\cos ^{2} \theta_{x x}}{\left(1-e \cos \theta_{x x}\right)^{2}}\right]} \\
& \frac{\partial \dot{\omega}_{x x i}}{\partial e}=\frac{f}{e} \sqrt{\frac{a}{\mu}}\left\{-\frac{1}{e} \sqrt{\frac{1}{1-e^{2}}\left[1+\frac{2\left(1-\cos ^{2} \theta_{x x}\right)}{1-e \cos \theta_{x x}}+\frac{1-\cos ^{2} \theta_{x x}}{\left(1-e \cos \theta_{x x}\right)^{2}}\right]}+\frac{1}{2}\left[\frac{-4 \cos \theta_{x x} \frac{d \cos \theta_{x x}}{d e}}{1+e \cos \theta_{x x}}-\right.\right. \\
& \left.\left.2 \frac{\left(1-\cos ^{2} \theta_{x x}\right)\left(e \frac{d \cos \theta_{x x}}{d e}+\cos \theta_{x x}\right)+\cos \theta_{x x} \frac{d \cos \theta_{x x}}{d e}}{\left(1+e \cos \theta_{x x}\right)^{2}}-\frac{2\left(1-\cos ^{2} \theta_{x x}\right)\left(e \frac{d \cos \theta_{x x}}{d e}+\cos \theta_{x x}\right)}{\left(1+e \cos \theta_{x x}\right)^{3}}\right] \sqrt{\frac{1-e^{2}}{1+\frac{2\left(1-\cos ^{2} \theta_{x x}\right)}{1-e \cos \theta_{x x}}+\frac{1-\cos ^{2} \theta_{x x}}{\left(1-e \cos \theta_{x x}\right)^{2}}}}\right\} \\
& \frac{\partial \dot{\omega}_{x x}}{\partial a}=\frac{f|\cos i|}{2 \sin i} \sqrt{\frac{1-e^{2}}{\mu a}} \frac{1}{\sqrt{1-e^{2} \cos ^{2} \omega}-e|\sin \omega|}
\end{aligned}
$$




$$
\begin{gathered}
\frac{\partial \dot{\Omega}_{x x}}{\partial e}=\frac{f|\cos i|}{\sin i} \sqrt{\frac{a}{\mu\left(1-e^{2}\right)}} \frac{1}{\sqrt{1-e^{2} \cos ^{2} \omega}-e|\sin \omega|}\left[\frac{1-e^{2}}{\sqrt{1-e^{2} \cos ^{2} \omega}-e|\sin \omega|}\left(\frac{e \cos ^{2} \omega}{\sqrt{1-e^{2} \cos ^{2} \omega}}+|\sin \omega|\right)-e\right] \\
\frac{\partial \dot{\Omega}_{x x}}{\partial i}=-f|\cos i| \sqrt{\frac{a\left(1-e^{2}\right)}{\mu}} \frac{1}{\left(\sqrt{1-e^{2} \cos ^{2} \omega}-e|\sin \omega|\right)}\left(\frac{1}{\cos i}+\frac{1}{\sin i \tan i}\right) \\
\frac{\partial \dot{\Omega}_{x x}}{\partial \omega}=\frac{f|\cos i|}{\sin i} \sqrt{\frac{a\left(1-e^{2}\right)}{\mu}} \frac{1}{\left(\sqrt{1-e^{2} \cos ^{2} \omega}-e|\sin \omega|\right)^{2}}\left(e \cos (\omega) \operatorname{sgn}(\sin \omega)-\frac{e^{2} \cos \omega \sin \omega}{\sqrt{1-e^{2} \cos ^{2} \omega}}\right)
\end{gathered}
$$

where

$$
\frac{d \cos \theta_{x x}}{d e}=\frac{\frac{1-\frac{3}{e^{2}}}{6 e^{2}}}{\sqrt{\left(\frac{1-e^{2}}{2 e^{3}}\right)^{2}+\frac{1}{27}}}\left\{\left[\frac{1-e^{2}}{2 e^{3}}+\sqrt{\left(\frac{1-e^{2}}{2 e^{3}}\right)^{2}+\frac{1}{27}}\right]^{\frac{1}{3}}+\left[\sqrt{\left(\frac{1-e^{2}}{2 e^{3}}\right)^{2}+\frac{1}{27}}-\frac{1-e^{2}}{2 e^{3}}\right]^{\frac{1}{3}}\right\}+\frac{1}{e^{2}}
$$

In order to apply Q-law, a method to calculate the above derivatives and minimize $d Q / d t$ at each point needs was implemented. Since the angles were not being changed very rapidly, updates to the thrust angles were made after $1 / 54^{\text {th }}$ of the current period of the transfer orbit, which amounted to the satellite moving approximately $6.66^{\circ}$ along its trajectory. This spacing was chosen to perform the assessment with practical speed of the trajectory calculations while ensuring that the angles generally converged to the correct values. When increasing the semimajor axis of the orbit, the in-plane angle, $\alpha$, was limited to the $-\pi$ to $\pi$ radians range and continuously increased within that range. The out-of-plane angle, $\beta$, was periodic within its range of $-\frac{\pi}{2}$ to $\frac{\pi}{2}$ radians. Any abrupt jumps in these angles indicated that the angle had converged to an incorrect value.

\section{References}

${ }^{1}$ Marian, V., Menudier, C., Thevenot, M., Vollaire, C., Verdier, J., Allard, B., "Efficient Design of Rectifying Antennas for Low Power Detection," 2011 International Microwave Symposium, Baltimore, MD, Jan. 2011.

${ }^{2}$ Lund, W., Nagel, A., Nalos. E., Rathjen, S., Woodcock, G., "Radially Symmetric Antenna Pattern Calculation," Boeing.

3 McSpadden, J., Mankins, J., "Space Solar Power Programs and Microwave Wireless Power Transmission Technology," IEEE Microwave Magazine, Dec. 2002.

${ }^{4}$ Fatemi, N., Pollard, H., Hou, H., Sharps, "Solar Array Trades Between Very High-Efficiency Multi-Junction and Si Space Solar Cells," IEEE PVSC, Anchorage Alaska, Sept. 17-22.

5 Petropoulos, A., "Refinements to the Q-Law for Low-Thrust Orbit Transfers," AIAA 2005-152, Copper Mountain Colorado, Jan. 23-27 2005. 